\title{
Three-Dimensional Cerebral Aneurysm Models for Surgical Simulation and Education-Development of Aneurysm Models with Perforating Arteries and for Application of Fenestrated Clips
}

\author{
Tatsuya Ishikawa1, Akio Morita2 ${ }^{2}$, Takanori Fukushima ${ }^{3}$, Hidenori Ono ${ }^{4}$ \\ ${ }^{1}$ Department of Surgical Neurology, Research Institute for Brain \& Blood Vessels, Akita, Japan \\ ${ }^{2}$ Department of Neurological Surgery, Nippon Medical School, Tokyo, Japan \\ ${ }^{3}$ Department of Neurosurgery, Duke University Medical Center \& Duke Raleigh Community Hospital \& West \\ Virginia University Medical Center, Durham, USA \\ ${ }^{4}$ Ono \& Co., Ltd., Tokyo, Japan \\ Email: teddyish@akita-noken.jp
}

Received 1 February 2014; revised 1 March 2014; accepted 8 March 2014

Copyright (C) 2014 by authors and Scientific Research Publishing Inc.

This work is licensed under the Creative Commons Attribution International License (CC BY).

http://creativecommons.org/licenses/by/4.0/

cC) (i) Open Access

\section{Abstract}

We modified a three-dimensional cerebral aneurysm model for surgical simulation and educational demonstration. Novel models are made showing perforating arteries arising around the aneurysm. Information about perforating arteries is difficult to obtain from individual radiological data sets. Perforators are therefore reproduced based on previous anatomical knowledge instead of personal data. Due to their fragility, perforating arteries are attached to the model using hard materials. At the same time, hollow models are useful for practicing clip application. We made a model for practicing the application of fenestrated clips for paraclinoid internal carotid aneurysms. Situating aneurysm models in the fissure of a brain model simulates the real surgical field and is helpful for educational demonstrations.

\section{Keywords}

Three-Dimensional Cerebral Aneurysm Model, Clipping Surgery, Simulation, Perforating Artery, Fenestrated Clip 


\section{Introduction}

Simulation of surgery is important in the field of vascular neurosurgeons, allowing the development of advanced surgical skills and compensation for a lack of clinical experience [1]. Advances in technology and materials have enabled the development of various kinds of neurovascular models. In particular, 3D reconstruction of information from radiological imaging is easily available using information technology, allowing the creation of $3 \mathrm{D}$ models.

In the endovascular era, young neurosurgeons have fewer opportunities to handle aneurysms through craniotomy by themselves, because a significant proportion of aneurysms are treated using endovascular coiling. Moreover, aneurysms that are unsuitable for coiling are generally complex and, thus, not very easy to approach for craniotomy and clipping. To compensate for this shortage in surgical experience, virtual reality simulations have recently been adapted to the field of neurosurgery.

Three-dimensional models of cerebral aneurysms have been used for preoperative simulation in surgical clipping [2] as well as endovascular coiling [3]. In clipping surgery, these models are useful for simulation, clarifying which kinds of aneurysm clips are appropriate based on the size and shape of the aneurysm, and how clips should be applied to eliminating the aneurysm from the circulation without obstructing or kinking the parent arteries [2]. However, these kinds of simulation lack important information regarding surrounding structures, particularly perforating vessels located adjacent to the aneurysm. In real surgery, perforating arteries should be preserved during surgical clipping to avoid cerebral ischemia and thus surgical complications [4]. However, fine surrounding structures, like the perforating vessels, are very difficult to reproduce in aneurysm models. One reason for this is that insufficient information on perforating arteries is obtained on radiological examinations such as 3D CTA. The other reason is that perforators are extremely fine vessels and are thus technically difficult to reproduce on aneurysm models. Nevertheless, it is possible to simulate the presence of perforating arteries using anatomical knowledge, and to apply theoretical perforating arteries (sometimes exaggerated) to the aneurysm model. We think that such models can be useful in educational demonstrations for young neurosurgeons. We therefore developed a 3D aneurysm model including virtual perforating vessels, and estimated the clinical importance of such models.

\section{Aneurysm Model}

We have made two kinds of aneurysm model for educational demonstrations to young neurosurgeons, allowing us to use these models to teach anatomical relationships between aneurysms and surrounding structures, and how to apply clips, including the direction of clip application and selection of clips (length and shape). The method for creating a 3D elastic and hard aneurysm model has been described elsewhere [2]. A hollow vascular model including an affected part is fabricated using a lamination technique together with supporting material to sustain the shape of the 3D model during fabrication by a precise lamination modeling machine. After the completion of fabrication by the lamination machine technique, the supporting material is removed. Perforating arteries are virtually designed on enhanced images from 3D CTA, referring to intra-operative information and the known vascular anatomy (Figure 1). Perforating arteries are created with a diameter of $0.5 \mathrm{~mm}$, slightly bigger than the real size. Moreover, the perforating arteries attached to the aneurysm model are very narrow in diameter. We have found that manipulation of hollow aneurysms easily breaks the perforating arteries. We therefore decided to make a hard aneurysm model with fine perforating arteries for demonstration purposes. At the same time, for simulation of manipulations, we made a hollow model without perforators.

More skill is required when using fenestrated clips to close the aneurysm, keeping the parent artery open. For aneurysms with a wide neck, we need to line the fenestrated clips up for complete closure. We have made an internal carotid aneurysm model at the paraclinoid segment, allowing us to practice the application of fenestrated clips (Figure 2).

Using these aneurysm models placed into brain models [5], we can simulate real aneurysm clipping. We can simulate and demonstrate the operative fields able to be obtained using a particular surgical approach, the direction of clip application, and the kind of clip to be used in the real surgery (Figure 3).

\section{Discussion}

When we make a model to establish a strict surgical plan on a particular patient, obtaining as much information 


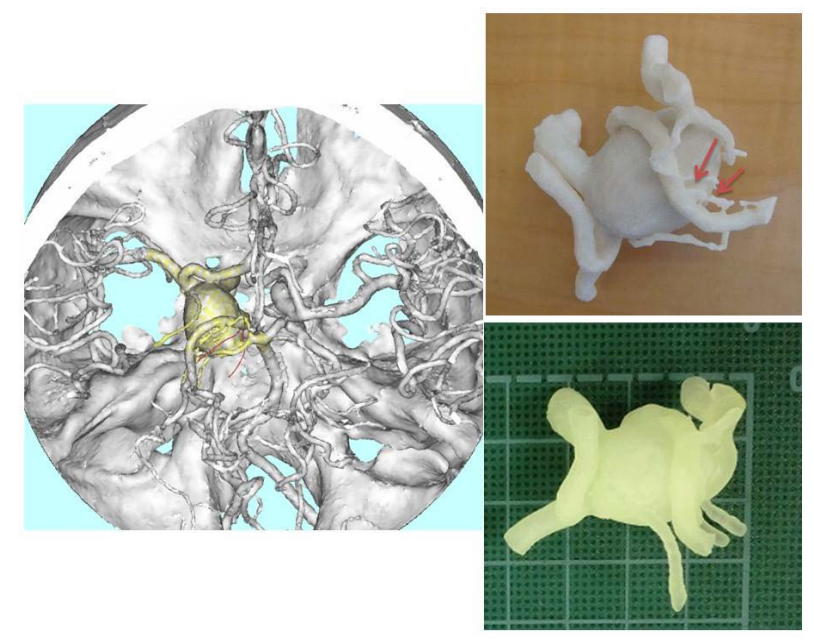

Figure 1. Images of an internal carotid artery aneurysm for perforator demonstration. Information on the imaginary perforating arteries (red curved line) arising nearby the aneurysm were added to the data-set from 3D CTA (left). The hard model made with imaginary perforating arteries (arrows) for demonstration purposes (upper right). The hollow model for clip application trials is made without perforators based on 3D CTA (lower right).

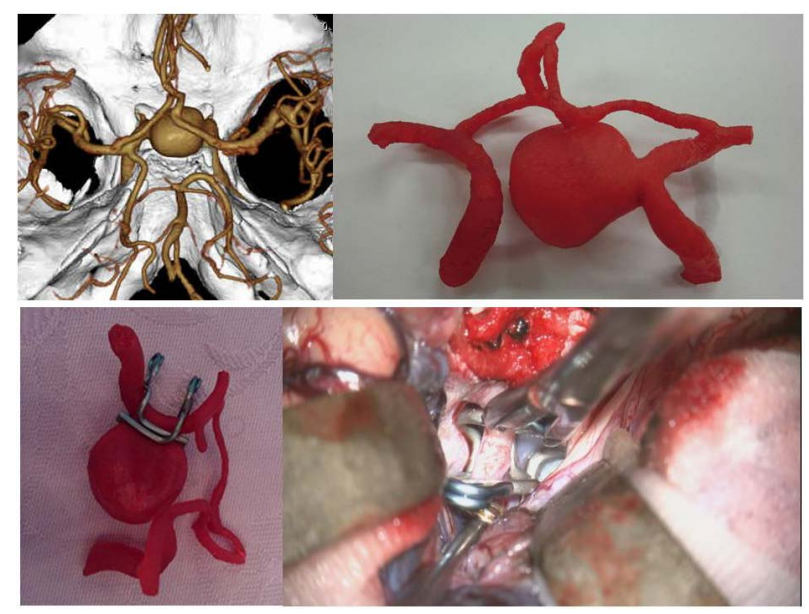

Figure 2. Images of the internal carotid artery aneurysm for practicing fenestration clip application. Based on the dataset from 3D CTA (upper left), we made a hard model of the aneurysm and the neighboring parent arteries (upper right). With the hollow model, the combination of the fenestrated clips is expected to be suitable for neck clipping (lower left). In the actual surgery, we used the combination of fenestrated clips in a very similar fashion. A temporary clip for the internal carotid artery, gold in color, is located behind the clips (lower right).

as possible from radiological examination is necessary. However, information is sometimes impossible to detect from the perforating arteries under radiological examination, as these vessels are too fine. However, individual anatomical information can be complemented by expectations based on the anatomical standard as well as personal experience. For educational purposes, where precise anatomical information is not always necessary, we have made various kinds of aneurysm models, showing how the aneurysm looks from a particular direction, and 

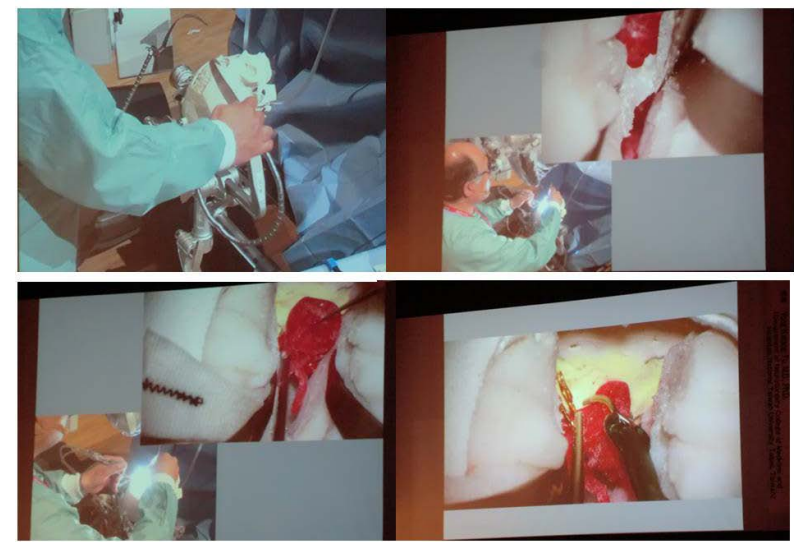

Figure 3. Demonstration of surgical clipping, showing how to use the surgical instruments and apply the clips, performed at an academic meeting (the 22nd Annual Meeting of the Conference on Neurosurgical Techniques and Tools, 2013). Macroscopic and/or microscopic views were projected onto the screen. The hollow model of the anterior communicating artery aneurysm is fixed in a KEZLEX A37 brain model (Ono, Tokyo, Japan). We explained how to use brain retractors (upper left), and showed how to dissect the interhemispheric fissure (upper right). The aneurysm neck was then dissected using the dissector (lower left) and secured with an aneurysm clip under proximal flow control by temporary clips (lower right).

where perforating arteries arise and run around the aneurysm, referring a lot of previous knowledge. Ideally, dissection of the perforating artery from the aneurysm and subsequent application of clips can be simulated on the models.

However, perforating arteries are too fine to reproduce on models and, moreover, cannot hold an anatomical position by themselves. Even if such vessels could be reproduced, they would be easily broken with even gentle manipulation. Perforators were therefore reproduced on the hard model with harder materials and thicker diameter than real size. In the hollow model, where information on perforating arteries was excluded, we could actually apply clips onto the model, as reported by Kimura et al. [2]. The knowledge obtained from hard models complements that obtained from being able to physically interact with hollow models.

Another situation for which we need to practice clip application is when we apply a combination of clip types, including fenestrated clips. We usually use fenestrated clips for complex-shaped and larger aneurysms [6], particularly those involving the paraclinoid portion of the internal carotid artery. We therefore made models of the internal carotid aneurysm at the paraclinoid portion, to demonstrate reconstructive clipping for younger neurosurgeons.

These models themselves are very educational, providing real 3D information on aneurysms. Even more helpful is when we can situate the aneurysm model in the fissure made by cerebral structures in a brain model like the KEZLEX A36 or A37 (Ono) [5]. With these models, young neurosurgeons can imagine how the aneurysm is located within the brain structure, and how to approach and eliminate the aneurysm using clips while avoiding injuries to perforating arteries. In addition, we have found that the combination of models is very useful for demonstrating how to use surgical instruments such as the brain retractor, clip applier, microscissors, and dissectors, in workshops on aneurysm surgery.

\section{Conclusion}

In conclusion, we have reported a minor but very useful modification of aneurysm models. This modification facilitates the educational application of models for microscopic aneurysm surgery.

\section{References}

[1] Mutoh, T., Ishikawa, T., Ono, H. and Yasui, N. (2010) A New Polyvinyl Alcohol Hydrogel Vascular Model (KEZLEX) 
for Microvascular Anastomosis Training. Surgical Neurology International, 1, 74.

[2] Kimura, T., Morita, A., Nishimura, K., Aiyama, H., Itoh, H., Fukaya, S., Sora, S. and Ochiai, C. (2009) Simulation of and Training for Cerebral Aneurysm Clipping with 3-Dimensional Models. Neurosurgery, 65, 719-725.

[3] Suzuki, Y., Fujitsuka, M. and Chaloupka, J.C. (2005) Simulation of Endovascular Neurointervention Using Silicone Models: Imaging and Manipulation. Neurologia Medico-Chirurgica, 45, 567-572.

[4] Yasui, N. and Nishimura, H. (2004) Surgical Treatment of Unruptured Intracranial Aneurysms over the Past 22 Years. Neurologia Medico-Chirurgica, 44, 155-161.

[5] Ishikawa, T., Yasui, N. and Ono, H. (2010) Novel Brain Model for Training of Deep Microvascular Anastomosis. Neurologia Medico-Chirurgica, 50, 627-629.

[6] Kato, Y., Sano, H., Imizu, S., Yoneda, M., Viral, M., Nagata, J. and Kanno, T. (2003) Surgical Strategies for Treatment of Giant or Large Intracranial Aneurysms: Our Experience with 139 Cases. Minimally Invasive Neurosurgery, 46, 339-343. 